\title{
Review
}

Journal of Innate

Immunity
J Innate Immun 2011;3:227-235

DOI: $\underline{10.1159 / 000324112}$
Received: November 24, 2010

Accepted after revision: January 6, 2011

Published online: March 22, 2011

\section{Vis-à-Vis in the NKC: Genetically Linked Natural Killer Cell Receptor/Ligand Pairs in the Natural Killer Gene Complex (NKC)}

\author{
Isabel Vogler Alexander Steinle \\ Institute for Molecular Medicine, Goethe University Frankfurt, Frankfurt am Main, Germany
}

\section{Key Words}

Cytotoxicity $\cdot$ Immune response $\cdot$ Vertebrates $\cdot$ Natural

killer cells $\cdot$ C-type lectin-like receptors $\cdot$ Natural killer gene complex receptor/ligand pairs in humans. Here, we provide a snapshot of the current knowledge on receptors of the NKRP1 family and their genetically linked CLEC2 ligands in mouse and man.

Copyright $\odot 2011$ S. Karger AG, Basel

\begin{abstract}
The natural killer gene complex (NKC) encodes several dozens of C-type lectin-like receptors that, in various ways, tune the reactivity of NK cells and other cytotoxic lymphocytes depending on the cellular environment. Among these are Ctype lectin-like receptors such as NKG2D, CD94/NKG2A and the murine Ly49 receptors that bind to cell surface glycoproteins of the major histocompatibility complex (MHC) class I family and thereby facilitate detection of stressed cells or cells exhibiting aberrant MHC class I expression. In contrast, NKRP1 receptors including the prototypic NK1.1 do not engage ligands with an MHC class--l-like fold, but rather interact with the likewise C-type lectin-like CLEC2 glycoproteins. Notably, CLEC2 and NKRP1 molecules not only share the same fold, but are also genetically linked in the NKC. Recent research efforts began to systematically elucidate the expression and function of the numerous NKRP1 and CLEC2 family members in rodents and revealed previously unnoticed corresponding
\end{abstract}

Natural killer (NK) cells are lymphocytes of the innate immune system capable of destroying aberrant and potentially harmful cells such as malignant or virus-infected cells [1-3]. Apart from their cytotoxic capacity, NK cells are also important producers of interferon (IFN)- $\gamma$ and other cytokines, thereby determining the course of immune responses [1-3]. Further, subsets of NK and NK-like cells have recently been described that appear to be involved in tissue homeostasis and tissuespecific immunosurveillance, illustrating the versatility of NK cells [4]. Reactivity of NK cells succumbs to the principles of 'missing self' and 'induced self' recognition relating to NK cell responses towards cells with deficient major histocompatibility complex (MHC) class I expression and with cell stress-induced expression of NKG2D ligands, respectively $[1,5]$. Elucidation of these principles largely benefited from the molecular description of NK cell receptors (NKRs) and their ligands, an

\section{KARGER}

Fax +41613061234

E-Mail karger@karger.ch

www.karger.com
(C) 2011 S. Karger AG, Basel

$1662-811 X / 11 / 0033-0227 \$ 38.00 / 0$

Accessible online at:

www.karger.com/jin
Dr. Alexander Steinle

Institute for Molecular Medicine, Goethe University Frankfurt am Main

Theodor-Stern-Kai 7

DE-60590 Frankfurt am Main (Germany)

Tel. +49696301 3700, E-Mail Alexander.Steinle@kgu.de 
approach that continues to unravel the immunobiology of NK cells beyond the recognition modes described by these two principles.

\section{The Natural Killer Gene Complex: A Gene Cluster of Immune-Related C-Type Lectin-Like Receptors}

In humans, many receptors governing the activity of NK cells are encoded in two distinct genomic regions: the leukocyte receptor complex (LRC) on chromosome 19 contains receptors of the immunoglobulin (Ig) superfamily such as killer Ig-like receptors (KIRs), leukocyte Ig-like receptors (LILRs) or NKp46 [6]. On the other hand, there is the NK cell gene complex (NKC) on chromosome 12 encoding for receptors of the C-type lectinlike superfamily $[3,7]$. For many of the NKR genes present in the human NKC, there are orthologues in the mouse NKC, a syntenic region on chromosome 6 . In contrast, for most genes of human Ig-like NKRs there are no corresponding loci on the mouse LRC on chromosome $7[3,6]$.

NKC-encoded C-type lectin-like receptors (CTLRs) were classified as group $\mathrm{V}$ receptors of the C-type lectinlike superfamily $[8,9]$. Typically, they are type II transmembrane glycoproteins with a single extracellular Ctype lectin-like domain (CTLD). Most of them occur as disulfide-linked homo- or heterodimers. They are also atypical C-type lectins, as their CTLD lacks the amino acids critical for $\mathrm{Ca}^{2+}$ binding, and therefore, lost the capacity to bind carbohydrates. Instead, most NKC-encoded CTLRs engage well-defined proteinaceous ligands $[3,8]$. An exception is the pattern recognition receptor dectin-1 (encoded by CLEC7A in the NKC) on myeloid cells that binds carbohydrate structures of fungal cell walls [10].

The overall structure of NKC-encoded CTLR starts with an amino-terminal cytoplasmic domain, followed by a single transmembrane domain, a stalk region of variable length and the CTLD with a carboxyterminal appendix [3]. Further, typical features of the CTLD are the so-called 'WIGL' motif, representing hydrophobic amino acids of the CTLD core structure (located in the central $\beta_{2}$ strand), and 6 conserved cysteines that further stabilize the CTLD by forming intramolecular disulfide bonds $[3,8]$.

NKC genes have been subgrouped into 'killer cell lectin-like receptor' (KLR) genes and 'C-type lectin receptor' (CLEC) genes, with KLR genes encoding for CTLR on NK cells, and CLEC genes designating all remaining genes [9]. Apart from this functional distinction, there are no clearcut structural or sequence-related hallmarks paralleling this classification. However, KLR and CLEC genes themselves can be further divided into subfamilies according to the sequence relatedness of the respective CTLD. Examples are the KLRA family encoding for mouse Ly49 receptors, the KLRC family encoding for NKG2 receptors (except NKG2D), the KLRB/F family encoding for NKRP1 receptors, and the CLEC2 family [9]. The latter includes the genes for $\mathrm{CD} 69$, for mouse Clr molecules and for the human CTLRs designated 'KACL', 'AICL' and 'LLT1' which are absent in rodents. These CLEC2 glycoproteins share unique sequence features in the CTLD such as a conserved 'FLkRy' motif in the carboxyterminal half of the $\alpha_{2}$ helix, a short L3 loop (preceding the $\beta_{3}$ strand), and a frequent lack of cysteines 4 and 5 that are highly conserved in other CTLRs [11-13]. Hitherto, structural data are only available for the CLEC2 family member CD69, suggesting that the FLkRy motif critically contributes to the dimerization of CLEC2 molecules [11, 12]. A short L3 loop in CLEC2 molecules may also render the disulfide bond between cysteines 4 and 5 dispensable that otherwise tacks the L2/L3 loop structure to the $\beta$-pleated sheet formed by the $\beta_{3}$ and $\beta_{4}$ strands $[11,12]$.

Notably, CLEC2 genes are intercalated with $K L R B / F$ genes in a subregion of both the human and the mouse NKC. In 2003, a seminal study reported that certain NKRP1 receptors modulate the activity of mouse NK cells by engaging members of the CLEC2 family, thereby critically advancing our understanding of the immunobiology of these two CTLR families [14]. Here, we review the current knowledge on NKRP1 and CLEC2 receptors, their expression and functional interaction.

\section{Mouse NKRP1 Receptors}

Over 30 years ago, first surface structures defining murine NK cells were described by means of monoclonal antibodies modulating the activity of NK cells $[15,16]$. One of these first NK cell-specific structures has been named 'NK1.1' and is expressed on NK cells of certain mouse strains such as C57BL/6 [15, 17]. Meanwhile, NK1.1 is known to represent an allelic variant encoded by the NKRP1 gene family member Nkrp1c [17]. NK1.1 as a prototypic member of the NKRP1 family has been widely used for the characterization and activation of mouse NK cells and, together with the Ly 49 receptors, was the 'founding member' of the NKC $[3,7]$.

Nkrplc is an activating receptor, as NK1.1-specific antibodies trigger degranulation and IFN- $\gamma$ secretion by mouse NK cells [18]. Activating signals by Nkrplc are 




Fig. 1. Genetic linkage of NKRP1 receptors and their C-type lectin-like ligands in the NKC of mouse and man. Schematic map of NKRP1 and CLEC2 genes in the centromeric subregion of the $\mathrm{NKC}$ of C57BL/6 mice (a) and in the telomeric subregion of the human NKC (b). Boxes represent genes of NKRP1 (dark grey) and CLEC2 gene families (light grey). Pseudogenes are hatched, genes encoding for other CTLRs are black. Arrows indicate transcriptional orientation and dashed lines indicate receptor/ligand interactions. Please note that for several genes evidence for expression of functional proteins, or even transcripts, is lacking. Maps are drawn to the respective scales. transduced through association with the immunoreceptor tyrosine-based activation motif (ITAM)-bearing adaptor FceR $\gamma \mathrm{I}$ facilitated by an arginine in the transmembrane region of Nkrplc [18]. Apart from Nkrplc, there are at least four additional NKRP1 receptors in mice: the inhibitory receptor Nkrplb (including the allelic variant Nkrpld in C57BL/6), as well as Nkrpla, Nkrplf and Nkrplg [3, 19, 20] (fig. 1). Nkrple appears to be a pseudogene as no Nkrple-derived cDNA encoding for a bona fide CTLD could be obtained [19]. Whereas most NKRP1 genes are almost monomorphic, both $N k r p 1 b$ and Nkrp1c exhibit a substantial polymorphism among various mouse strains [19].

Insights into the biology of Nkrpl receptors apart from Nkrplc were long hampered by the lack of specific reagents. Recently, Aust and colleagues [20] reported a novel set of monoclonal antibodies specifically detecting Nkrpla, Nkrpld and Nkrplf in C57BL/6 mice. Using these reagents, Nkrpla and Nkrplf were detectable on virtually all NK cells derived from spleen and bone marrow albeit at low levels, whereas Nkrpld is expressed by only about half of splenic NK cells [20]. In contrast to Nkrplc, Nkrpla and Nkrplf were found to be expressed in a highly NK-specific manner, as resting and activated $\mathrm{T}$ cells including NK1.1 $1^{+} \mathrm{T}$ cells were completely negative [20]. An earlier report found abundant Nkrplf transcripts in bone marrow-derived dendritic cells (DCs), but corresponding surface expression of Nkrplf remains to be confirmed [21]. An arginine residue in the transmembrane domains of both Nkrpla and Nkrplf implied an activating function analogous to Nkrplc, but contrary to expectations, antibody-mediated cross-linking of Nkrpla and Nkrplf did not enhance NK cell effector functions such as cytotoxicity and cytokine secretion [20]. In Nkrplb, there is a functional immunoreceptor tyrosine-based inhibitory motif (ITIM) at the amino terminus of the cytoplasmic region mediating the reported inhibitory function $[14,22]$. An ITIM-like motif is also present in the cytoplasmic portion of Nkrplg, likewise suggesting an inhibitory function, but thus far, Nkrplg expression could not be assessed due to the lack of a specific detection reagent. 


\section{Mouse NKRP1 Receptors Engage Genetically Linked Clr Molecules}

Initially, ligands of Nkrp1 receptors were identified in an approach employing reporter cells with Nkrpl hybrid receptors and soluble Nkrp1 multimers [14]. Intriguingly, Nkrpld and Nkrplf were reported to engage CTLRs of the CLEC2 family encoded in close genetic linkage to their respective receptors [14]. Nkrplf was shown to specifically interact with Clr-g, whereas allelic variants of Nkrplb specifically bind to Clr-b [14, 23]. Accordingly, ectopic overexpression of Clr-b reduced the susceptibility of cell lines to cytolysis by interleukin (IL)-2-activated NK cells isolated from C57BL/6 mice [14]. Physiological expression of Clr-b had only a minor effect on NK cell cytolysis, indicating that expression levels critically determine the functional impact of Nkrplb/Clr-b interactions [20, 23]. With regard to functional consequences of Nkrplf/Clr-g interaction, a costimulatory role has been suggested during T-cell activation with activated $\mathrm{T}$ cells upregulating Clr-g that may interact with Nkrplf on bone marrow-derived DCs [21]. These were the first reports showing that certain C-type lectin-like NKRs encoded in the NKC specifically interact with likewise C-type lectin-like ligands shedding light on the previously neglected group of NKCencoded Clr proteins. Thus far, no ligands have been described for other Nkrpl family members including Nkrplc, and it remains to be determined whether these ligands are likewise recruited from the Clr cohort.

\section{Mouse Clr Molecules}

In mice, there are at least 9 genes of the CLEC2 family (CD69, Clr-a to Clr-h). Apart from CD69, 7 of these CLEC2 genes potentially encode for functional Clr molecules, whereas $\mathrm{Clr}$-e appears to be a pseudogene as its coding sequence contains numerous stop codons (fig. 1) $[19,24]$. CLEC2 genes are of low polymorphism and map between the loci of Nkrp1a and CD69 to the centromeric portion of the mouse NKC $[7,19,24]$. Up to now, there is rather limited knowledge on the expression and function of most $\mathrm{Clr}$ proteins. The best characterized family member by far is Clr-b: Clr-b is expressed by almost all hematopoietic cells and was shown to specifically bind to the inhibitory receptor Nkrplb/d [14, 23]. Clr-b exhibits a broad tissue expression, similar to that of MHC class I, and Clr-b expression has also been specifically reported for osteoclasts [25]. Accordingly, adult Clr-b-deficient mice suffer from osteopenia, but do not exhibit apparent immune function defects [25]. Notably, Clr-b downregulation has been observed for many tumor cell lines, thus favoring NK cell-mediated lysis [23]. Very recently, genotoxic stress has been shown to negatively modulate $\mathrm{Clr}-\mathrm{b}$ expression, an observation further promoting the concept of MHC-independent regulation of NK cell function by NKRP1 receptors [26]. In this regard, Clr-b appears inversely regulated to ligands of the activating receptor NKG2D that are inducibly expressed upon genotoxic stress $[1,5]$. Likewise, in contrast to NKG2D ligands, infection with rat cytomegalovirus (RCMV) is associated with a drastic drop in levels of rat Clr-b transcripts. RCMV-infected cells maintain inhibitory signals by expression of the RCMV-encoded Clr-b surrogate RCTL (or RCMV C-type lectin) engaging Nkrplb [27].

Apart from Clr-b, expression and function of other $\mathrm{Clr}$ genes are barely characterized: Clr-g transcripts have been detected in bone marrow-derived DCs, macrophages, lymphokine-activated killer cells and activated T cells [14, $21,24]$. Clr-f transcripts have been reported for IL-2-activated NK cells and some tissues [24]. No data are available for tissue expression of $\mathrm{Clr}-a, C l r-c, C l r-d$ and $C l r-h$, respectively. In rats, similar sets of NKRP1 and CLEC2 genes have been described, including the interaction between the corresponding Nkrp1 receptors and Clr molecules [28, 29]. Obviously, studies so far have provided rather basic information on expression and receptor/ligand interaction of Nkrpl receptors and Clr molecules, and we are just at the beginning of the quest for a better understanding of the immunological relevance of these CTLRs.

\section{NKR-P1A, an Inhibitory NKRP1 Receptor in Humans}

The only generally recognized human homologue of the rodent Nkrp1 family is NKR-P1A/CD161 based on an amino acid sequence identity of $46 \%$ to Nkrpld. NKR$\mathrm{P} 1 \mathrm{~A}$ is encoded by the gene KLRB1 located at the telomeric end of the human NKC [18] (fig. 1) and expressed on the majority (approximately 73-97\%) of human peripheral blood NK cells [30]. NKR-P1A is also present on about $25 \%$ of peripheral blood $\mathrm{T}$ cells, preferentially on those with a memory phenotype [30]. Notably, the majority of intraepithelial $\mathrm{T}$ cells in the small intestine expresses NKR-P1A [31], and recently, all IL-17-producing T-cell subsets were shown to share surface expression of NKRP1A due to the activity of the transcription factor 'retinoic acid-related orphan receptor C' (RORC) [32]. NKR$\mathrm{P} 1 \mathrm{~A}$ is considered an inhibitory receptor, although there are some conflicting data for NKR-P1A-expressing $\mathrm{T}$ 
Fig. 2. Interaction of human NKRP1 receptors with their genetically linked ligands of the CLEC2 family. Schematic drawing of NKRP1 receptors NKR-P1A, NKp80 and NKp65 and their respective ligands together with some characteristics. The dissociation constant $\left(\mathrm{K}_{\mathrm{d}}\right)$ was not detectable (n.d.) for NKR-P1A/LLT1 interaction.

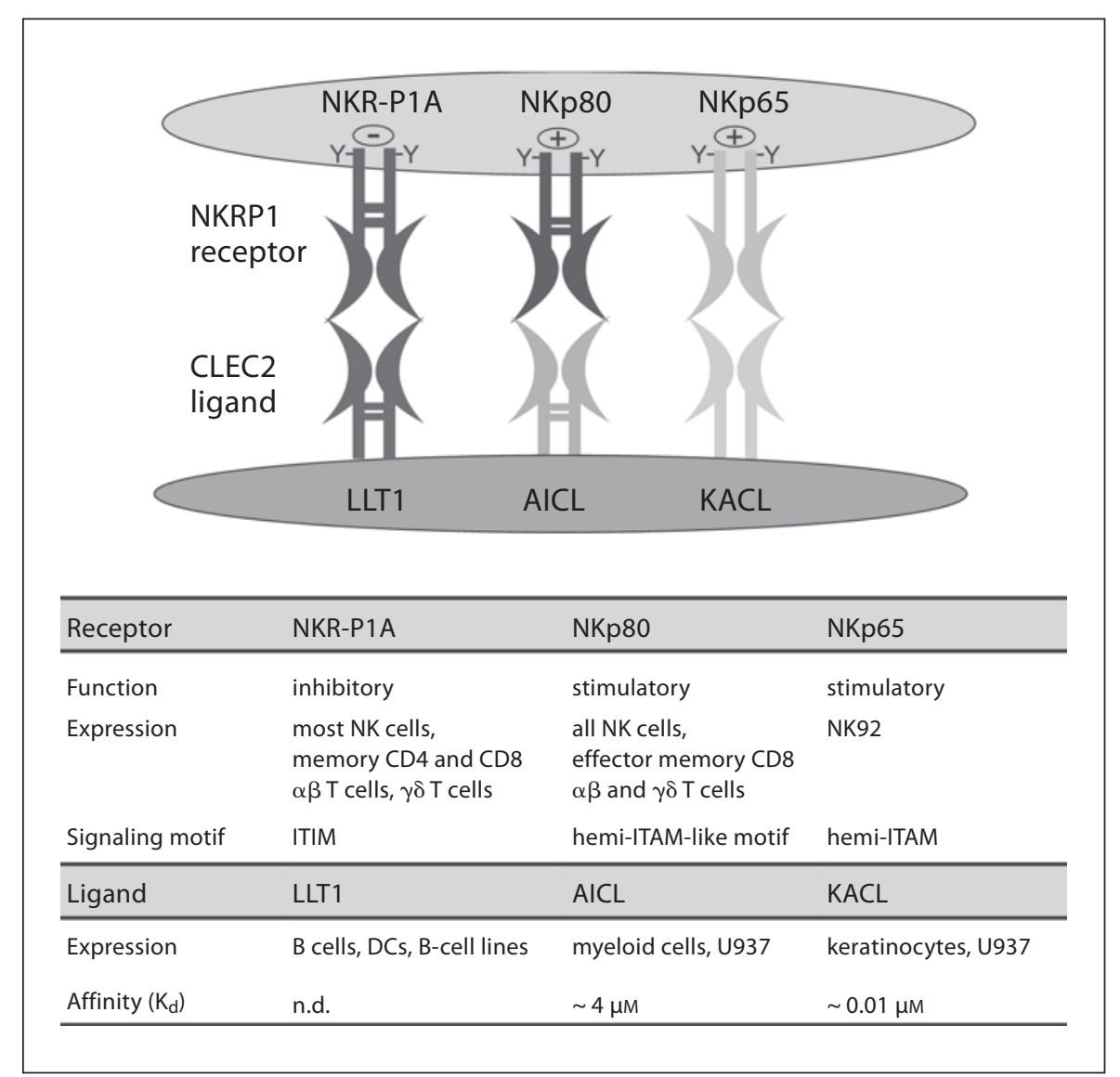

cells, as discussed elsewhere [33]. For NK cells, NKR-P1A engagement inhibits cytotoxicity and cytokine secretion $[34,35]$. Accordingly, there is a noncanonical ITIM sequence at the amino terminus of the NKR-P1A cytoplasmic domain similarly to mouse Nkrplb. Acid sphingomyelinase has also been reported as an intracellular interaction partner of NKR-P1A, with NKR-P1A cross-linking leading to acid sphingomyelinase activation and ceramide production, as well as subsequent downstream activation of Akt and proliferation [36]. It is currently unclear how this mechanism contributes to the immunological function of NKR-P1A.

In view of the findings of genetically linked Nkrp1-Clr receptor/ligand pairs in mice, it was suggested that NKRP1A may interact with the CLEC2 family member LLT1 that is encoded by the CLEC2D gene approximately $60 \mathrm{~kb}$ apart from the KLRB1 gene in the human NKC $[3,37]$. This supposition was promptly verified by two independent studies reporting LLT1 as the natural ligand for human NKR-P1A (fig. 2) [34, 35]. Functional analysis of the LLT1/NKR-P1A interaction demonstrated that ectopic expression of LLT1 on target cells reduces NK cell-mediated cytotoxicity and partially inhibits IFN- $\gamma$ secretion $[34,35]$. These results confirmed an inhibitory function of NKR-P1A, as suggested by earlier antibody-based experiments [30]. The capacity of LLT1 to inhibit NK cell function may also be exploited by tumors to escape from NK cell attack, as the expression of LLT1 was described for malignant glioma impairing NK cell cytotoxicity [38].

Endogenously, LLT1 is expressed by various B-cell lines and upregulated on activated B cells, as well as on plasmacytoid and myeloid DCs stimulated with Toll-like receptor ligands [33]. Based on these results, it was proposed that LLT1 upregulation in the course of Toll-like receptor-mediated DC maturation may contribute to the observed resistance of mature DCs towards cytolysis by NK cells [33]. Similar mechanisms may apply for activated B cells introducing LLT1 as a potential regulator of the interaction of NK cells with DCs and B cells [33].

The function of NKR-P1A for T-cell responses remains unclear. One study reported that NKR-P1A interaction stimulates IFN- $\gamma$ secretion by polyclonal $\mathrm{T}$ cells 
[34], whereas a second study found no modulation of effector responses by CD 4 and CD8 T cells upon NKR-P1A engagement apart from an occasional reduction in tumor necrosis factor (TNF) secretion by CD8 T cells [33]. Other reports implicated NKR-P1A in the transendothelial migration of resting CD4 T cells and $\gamma \delta$ T cells [39].

\section{NKp80 and NKp65 - Activating NKRP1 Receptors in Humans}

Whereas both activating and inhibitory Nkrpl receptors were described in rodents, NKR-P1A was considered the only human Nkrp1 homologue without an activating counterpart. However, more recent studies indicate that the receptor NKp80 encoded by the gene KLRF1 (in the same subregion of the human NKC) (fig. 1) [40,41] may represent the activating counterpart to NKR-P1A. In 2001, NKp80 was identified during a search for novel surface molecules specifically expressed by human NK cells and characterized as disulfide-linked homodimeric CTLR on virtually all NK cells [41]. Antibody-mediated cross-linking of NKp80 triggered $\mathrm{Ca}^{2+}$ influx as well as redirected NK cell cytotoxicity, where cytotoxicity correlated with the coexpression of the activating receptor NKp46 [41]. In direct cytolysis assays, NKp80 apparently contributed to the NK cell cytotoxicity against phytohemagglutinin-activated T-cell blasts, suggesting that the latter express an NKp80 ligand [41].

Apart from NK cells, there is also surface NKp80 on subpopulations of cytotoxic T cells [41-43]: NKp80 is present to a variable extent on human $\alpha \beta C D 8 \mathrm{~T}$ cells (mean 22\%, range 5-55\%) and $\gamma \delta \mathrm{T}$ cells (mean 26\%, range $6-65 \%)[42,43]$. On $\alpha \beta C D 8 \mathrm{~T}$ cells, NKp80 is strictly confined to the effector memory and terminal effector subsets that exhibit an NK cell-like phenotype and costimulates their T-cell receptor-activated effector responses such as cytotoxicity and IFN- $\gamma$ secretion [43]. NKp80 is also expressed on some T cells and virtually all NK cells of macaques where it likewise exerts an activating function $[44,45]$, whereas no NKp80-related receptors were identified in rodents (approximately $28 \%$ amino acid sequence identity between NKp80 and mouse Nkrp1 receptors). Signaling events subsequent to NKp80 engagement have remained unknown. In contrast to the activating mouse NKRP1 receptor Nkrplc and most other activating human NKRs, the transmembrane domain of NKp80 contains no charged amino acids, and therefore, an association with ITAM-bearing signaling adaptors such as FceRI $\gamma, \mathrm{CD} 3 \zeta$ or DAP12 appears unlikely.
Recently, a novel CTLR most closely related to NKp 80 and encoded in proximity to the KLRF1 locus by the $K L R F 2$ gene was reported (fig. 1) [46]. According to its calculated molecular mass and in analogy to NKp80, this receptor was termed 'NKp65' and characterized as a nondisulfide-linked homodimeric cell surface receptor [46]. Like NKp80, NKp65 triggers NK cell cytotoxicity in assays of redirected cytolysis. But unlike NKp80, NKp65 is not detectably expressed on human peripheral blood NK or T cells, although NKp65 cDNA was originally cloned from IL-2/IL-12-stimulated peripheral blood NK cells [46]. So far, significant surface expression of NKp65 has been noted only for the NK cell line NK92 and its derivative NK92MI [46], and hence, cells physiologically expressing NKp65 and the determining factors of NKp65 expression in vivo await elucidation. Interestingly, the amino terminus of the NKp65 cytoplasmic domain harbors a tyrosine-containing sequence motif that matches the consensus of a so-called 'hemi-ITAM' (i.e. DEDGYxxL) as deduced from Syk-recruiting motifs of dectin-1/CLEC7A and CLEC-2/CLEC1B [47] (please note that CLEC-2 is the common name of the CLEC1B gene product, but is not a member of the CLEC2 family of proteins). Both dectin-1 and CLEC-2 are also NKC-encoded CTLRs, but their expression is restricted to certain myeloid cells such as macrophages (dectin-1) or platelets (CLEC-2). Mutation of the amino-terminal tyrosine 7 in NKp65 abrogated the capacity of NKp65 to trigger cytotoxicity [46], supporting the notion that NKp65 may signal via a hemi-ITAM and Syk kinase. Correspondingly, we recently also identified a hemi-ITAM-like sequence in the cytoplasmic domain of NKp80 that becomes phosphorylated at tyrosine 7 and is critically involved in NKp80 signaling [48].

\section{NKp80 Engages AICL Promoting the Cross-Talk between NK and Myeloid Cells}

Based on the reports that mouse Nkrp1 receptors are genetically linked with their ligands recruited from the Clr/CLEC2 protein family $[14,23]$, human members of the CLEC2 family encoded in the proximity to the KLRF1 locus were assayed for binding to NKp80. In fact, it turned out that the CTLR AICL encoded by the CLEC2B gene acts as a natural ligand of NKp80 (fig. 2) [42]. $C L E C 2 B$ and KLRF1 genes are located just $7 \mathrm{~kb}$ apart in a tail-to-tail orientation (fig. 1). AICL transcripts are broadly detectable in peripheral blood granulocytes, monocytes and lymphocytes, and transcript levels are 
increased upon treatment with phorbol myristate acetate (PMA) $[42,49]$. AICL-specific antibodies revealed AICL expression on myeloid cells such as monocytes, macrophages and granulocytes [42]. Whereas NKG2D ligands are broadly present on many tumor cell lines, AICL was only found on a few malignant cell lines of myeloid origin $[42,50]$. A recent study also reported AICL expression by some non-hematopoietic cell lines and liver cancer specimens [51].

In functional terms, NKp80/AICL interaction promotes cytolysis of malignant, AICL-expressing cells by NK cells and effector memory CD8 T cells [42, 43, 51]. AICL expression on monocytes is enhanced by lipopolysaccharide and, in the presence of lipopolysaccharide or inflammatory cytokines, NKp80/AICL interaction stimulates an activating cross-talk between NK cells and monocytes as well as between effector memory CD8 T cells and macrophages [42, 43, 52]. Hence, this NKC-encoded receptor/ligand pair may promote immune responses in early phases of infection or during chronic inflammation. Since there are no sequence homologues for both AICL and NKp80 in rodents, investigating the in vivo function of this receptor/ligand pair remains challenging. However, there is first evidence that the stimulation of immune responses via NKp80/AICL interaction is targeted by pathogens: the immune evasion gene $\mathrm{K} 5$ of Kaposi's sarcoma-associated herpesvirus has been reported to downregulate not only the NKG2D ligands MICA and MICB, but also AICL [53]. It remains to be shown whether other viruses that frequently interfere with NK cell-mediated immunosurveillance such as adenoviruses or CMV likewise evolved strategies to intercept NKp80/AICL interaction.

\section{NKp65 Engages KACL Facilitating the Immunorecognition of Keratinocytes}

The latest addition to the human CLEC2 gene family is CLEC2A, a gene localized between KLRF2 and CLEC12A in the human NKC (fig. 1) $[13,46]$. The delayed description of CLEC2A and its product KACL is likely due to its unusual and restricted expression pattern that is fundamentally different from the other human CLEC2 genes CLEC2B (encoding for AICL), CD69/CLEC2C and CLEC2D (LLT1). Whereas transcripts of the latter are abundantly present in hematopoietic cells of the peripheral blood and detectable in most tissues, CLEC2A transcripts are mostly restricted to human skin, with only very low levels detectable in some other tissues including

NKRP1 Receptors and Their Ligands bone marrow, spleen and gonadal tissues $[13,46]$. Like many other CTLR genes, CLEC2A gives rise to several alternatively spliced transcripts with only one transcript (designated 'CLEC2A1') yielding a glycoprotein detectable at the cell surface (i.e. KACL) [13]. Again, in contrast to other human CLEC2 family members, KACL homodimers are not disulfide linked and lack both cysteines 4 and 5 of the CTLD [46]. Notably, both cysteines are also absent from mouse Clr-b and Clr-g. Similarly to AICL, pronounced cell surface expression of KACL was only observed for the myeloid cell line U937, whereas KACL was not detectable on non-hematopoietic cell lines [13, 46]. KACL is broadly expressed by freshly isolated keratinocytes in line with the skin-associated KACL transcripts. Reports on KACL expression by activated peripheral blood lymphocytes are inconsistent $[46,54]$.

Ectopic expression of KACL rendered cells susceptible to cytotoxicity by NK92MI cells and suggested the existence of an activating KACL receptor. Identification of NKp65 as KACL receptor was guided by the principle of genetic linkage, also applying to the respective genes KLRF2 and CLEC2A that localize in a tail-to-tail orientation only $2.5 \mathrm{~kb}$ apart next to the gene pair KLRF1/ CLEC2B (fig. 1). Similarly to KACL, but in contrast to other human NKRP1 receptors, NKp65 homodimers are not disulfide linked, and the affinity of the NKp65/KACL interaction is considerably higher $\left(\mathrm{K}_{\mathrm{d}}\right.$ approximately 12 $\mathrm{nM}$ ) [46] than the affinity of the NKp80-AICL interaction $\left(\mathrm{K}_{\mathrm{d}}\right.$ approximately $\left.4 \mu \mathrm{M}\right)$ [42] or of the NKR-P1A-LLT1 interaction $\left(\mathrm{K}_{\mathrm{d}}\right.$ above detection range; our unpubl. observations). Apart from the interactions between adjacently encoded NKRP1 and CLEC2 receptors, no additional crisscross binding was detected between these NKRP1 and CLEC 2 receptors by two studies $[46,55]$, whereas a third study also reported an interaction between NKRP1A and KACL (alias PILAR) [54]. In functional terms, NKp65/KACL interaction readily triggers cytotoxicity and IFN- $\gamma$ secretion by NKp65-expressing NK92MI cells and also stimulates effector responses of the latter towards keratinocytes [46]. Hence, NKp65 facilitates the dedicated immunorecognition of keratinocytes and may fulfill a yet unappreciated role in the immunosurveillance of human skin. Here, characterization of NKp65expressing cells will be a key factor in evaluating this notion when appropriate NKp65-specific antibodies become available. Similarly to NKp80 and AICL, no related sequences for NKp65 and KACL are described for rodents, although genes encoding for apparent homologues of NKp65 and KACL are present in genomes of chimpanzee, rhesus macaque and cow, respectively.

J Innate Immun 2011;3:227-235 


\section{Concluding Remarks}

Emerging evidence documents that the human NKC also encodes for several genetically coupled receptor/ligand pairs recruited from members of the NKRP1 and CLEC2 families as originally reported for the mouse NKC. Based on sequence comparisons, NKR-P1A and its ligand LLT1 were considered the only human homologues of mouse Nkrpl and Clr molecules, respectively. However, we would like to argue that NKp80/AICL and NKp65/KACL should likewise be grouped into this class of receptor/ligand pairs based on the following characteristics: (1) tight genetic linkage of receptor and ligand; (2) CLEC2-specific sequence hallmarks shared between LLT1, AICL, KACL and mouse Clr molecules as outlined above, and (3) amino-terminal immunoreceptor tyrosine-based signaling motifs shared between NKp80, NKp65, NKR-P1A and most mouse Nkrp1 receptors. At the moment, we are just at the beginning to understand the immunological relevance of these NKRP1/CLEC2 receptor/ligand pairs, and therefore, it is difficult to assess whether the respective human and mouse NKRP1/ CLEC2 receptor pairs also fulfill similar functions in vivo. Thus far, most data are available for mouse Nkrplb/d engaging $\mathrm{Clr}-\mathrm{b}$, which has been interpreted as a second layer of 'missing self' recognition independent of MHC class I. Whereas the rather ubiquitous expression of Clr$\mathrm{b}$, and its downregulation by RCMV, as well as genotoxic stress appear in support of this hypothesis, our knowledge of other members of the NKRP1 and CLEC2 families, their expression, ligands and function is too fragmentary at this point. Restricted tissue expression of CLEC2 family members such as AICL and LLT1 (hematopoietic cells), KACL (skin) and certain members of the mouse Clr family [24] (our unpubl. observations) also raise the possibility that certain NKRP1/CLEC2 receptor/ligand systems may specifically be involved in the regulatory cross-talk between immune cells or in maintaining tissue-specific immunity. Future studies on these peculiar genetically linked receptor/ligand pairs can be expected to broaden our view on NK cell biology.

\section{Acknowledgements}

Our work on CTLRs is supported by grants from the Deutsche Krebshilfe and from the Deutsche Forschungsgemeinschaft.

\section{References}

1 Raulet DH, Vance RE: Self-tolerance of natural killer cells. Nat Rev Immunol 2006;6: 520-531.

$>2$ Vivier E, Tomasello E, Baratin M, Walzer T, Ugolini S: Functions of natural killer cells. Nat Immunol 2008;9:503-510.

-3 Yokoyama WM, Plougastel BF: Immune functions encoded by the natural killer gene complex. Nat Rev Immunol 2003;3:304-316.

$\checkmark 4$ Di Santo JP: Natural killer cells: diversity in search of a niche. Nat Immunol 2008;9:473475.

5 Bryceson YT, Long EO: Line of attack: NK cell specificity and integration of signals. Curr Opin Immunol 2008;20:344-352.

$\checkmark 6$ Kelley J, Walter L, Trowsdale J: Comparative genomics of natural killer cell receptor gene clusters. PLoS Genet 2005;1:129-139.

$>7$ Yokoyama WM, Seaman WE: The Ly-49 and NKR-P1 gene families encoding lectin-like receptors on natural killer cells: the NK gene complex. Annu Rev Immunol 1993;11:613635.

$>8$ Zelensky AN, Gready JE: The C-type lectinlike domain superfamily. FEBS J 2005;272: 6179-6217.

$>9$ Hao L, Klein J, Nei M: Heterogeneous but conserved natural killer receptor gene complexes in four major orders of mammals. Proc Natl Acad Sci USA 2006;103:3192-3197.
10 Reid DM, Gow NA, Brown GD: Pattern recognition: recent insights from Dectin-1. Curr Opin Immunol 2009;21:30-37.

11 Llera AS, Viedma F, Sanchez-Madrid F, Tormo J: Crystal structure of the C-type lectinlike domain from the human hematopoietic cell receptor CD69. J Biol Chem 2001;276: 7312-7319

12 Natarajan K, Sawicki MW, Margulies DH, Mariuzza RA: Crystal structure of human CD69: a C-type lectin-like activation marker of hematopoietic cells. Biochemistry 2000; 39:14779-14786.

13 Spreu J, Kienle EC, Schrage B, Steinle A: CLEC2A: a novel, alternatively spliced and skin-associated member of the NKC-encoded AICL-CD69-LLT1 family. Immunogenetics 2007;59:903-912.

14 Iizuka K, Naidenko OV, Plougastel BF, Fremont DH, Yokoyama WM: Genetically linked C-type lectin-related ligands for the NKRP1 family of natural killer cell recep tors. Nat Immunol 2003;4:801-807.

15 Glimcher L, Shen FW, Cantor H: Identification of a cell-surface antigen selectively expressed on the natural killer cell. J Exp Med 1977;145:1-9.
16 Chambers WH, Vujanovic NL, DeLeo AB, Olszowy MW, Herberman RB, Hiserodt JC: Monoclonal antibody to a triggering structure expressed on rat natural killer cells and adherent lymphokine-activated killer cells. J Exp Med 1989;169:1373-1389.

-17 Carlyle JR, Mesci A, Ljutic B, Belanger S, Tai LH, Rousselle E, Troke AD, Proteau MF, Makrigiannis AP: Molecular and genetic basis for strain-dependent NK1.1 alloreactivity of mouse NK cells. J Immunol 2006;176: 7511-7524.

18 Arase N, Arase H, Park SY, Ohno H, Ra C, Saito T: Association with FcRgamma is essential for activation signal through NKR-P1 (CD161) in natural killer $(\mathrm{NK})$ cells and NK1.1+ T cells J Exp Med 1997;186:1957-1963.

19 Carlyle JR, Mesci A, Fine JH, Chen P, Belanger S, Tai LH, Makrigiannis AP: Evolution of the Ly49 and Nkrp1 recognition systems. Semin Immunol 2008;20:321-330.

20 Aust JG, Gays F, Mickiewicz KM, Buchanan E, Brooks CG: The expression and function of the NKRP1 receptor family in C57BL/6 mice. J Immunol 2009;183:106-116.

-21 Tian W, Nunez R, Cheng S, Ding Y, Tumang J, Lyddane C, Roman C, Liou HC: C-type lectin OCILRP2/Clr-g and its ligand NKRP1f costimulate $\mathrm{T}$ cell proliferation and IL-2 production. Cell Immunol 2005;234:39-53. 
-22 Ljutic B, Carlyle JR, Filipp D, Nakagawa R, Julius M, Zuniga-Pflucker JC: Functional requirements for signaling through the stimulatory and inhibitory mouse NKR-P1 (CD161) NK cell receptors. J Immunol 2005; 174:4789-4796.

-23 Carlyle JR, Jamieson AM, Gasser S, Clingan CS, Arase H, Raulet DH: Missing self-recognition of Ocil/Clr-b by inhibitory NKR-P1 natural killer cell receptors. Proc Natl Acad Sci USA 2004;101:3527-3532.

24 Plougastel B, Dubbelde C, Yokoyama WM: Cloning of Clr, a new family of lectin-like genes localized between mouse Nkrpla and Cd69. Immunogenetics 2001;53:209-214.

-25 Kartsogiannis V, Sims NA, Quinn JM, Ly C, Cipetic M, Poulton IJ, Walker EC, Saleh H, McGregor NE, Wallace ME, Smyth MJ, Martin TJ, et al: Osteoclast inhibitory lectin, an immune cell product that is required for normal bone physiology in vivo. J Biol Chem 2008;283:30850-30860.

-26 Fine JH, Chen P, Mesci A, Allan DS, Gasser S, Raulet DH, Carlyle JR: Chemotherapy-induced genotoxic stress promotes sensitivity to natural killer cell cytotoxicity by enabling missing-self recognition. Cancer Res 2010; 70:7102-7113.

-27 Voigt S, Mesci A, Ettinger J, Fine JH, Chen P, Chou W, Carlyle JR: Cytomegalovirus evasion of innate immunity by subversion of the NKR-P1B:Clr-b missing-self axis. Immunity 2007;26:617-627.

28 Flornes LM, Nylenna O, Saether PC, Daws MR, Dissen E, Fossum S: The complete inventory of receptors encoded by the rat natural killer cell gene complex. Immunogenetics 2010;62:521-530.

-29 Kveberg L, Dai KZ, Westgaard IH, Daws MR, Fossum S, Naper C, Vaage JT: Two major groups of rat NKR-P1 receptors can be distinguished based on chromosomal localization, phylogenetic analysis and Clr ligand binding. Eur J Immunol 2009;39:541-551.

- 30 Lanier LL, Chang C, Phillips JH: Human NKR-P1A. A disulfide-linked homodimer of the C-type lectin superfamily expressed by a subset of NK and T lymphocytes. J Immunol 1994;153:2417-2428.

- 31 Iiai T, Watanabe H, Suda T, Okamoto H, Abo T, Hatakeyama K: CD161+ T (NT) cells exist predominantly in human intestinal epithelium as well as in liver. Clin Exp Immunol 2002;129:92-98.

- 32 Maggi L, Santarlasci V, Capone M, Peired A, Frosali F, Crome SQ, Querci V, Fambrini M, Liotta F, Levings MK, Maggi E, Cosmi $\mathrm{L}$, et al: CD161 is a marker of all human IL17-producing T-cell subsets and is induced by RORC. Eur J Immunol 2010;40:21742181.

33 Rosen DB, Cao W, Avery DT, Tangye SG, Liu YJ, Houchins JP, Lanier LL: Functional consequences of interactions between human NKR-P1A and its ligand LLT1 expressed on activated dendritic cells and B cells. J Immunol 2008;180:6508-6517.
4 Aldemir H, Prod'homme V, Dumaurier MJ, Retiere C, Poupon G, Cazareth J, Bihl F, Braud VM: Cutting edge: lectin-like transcript 1 is a ligand for the CD161 receptor. J Immunol 2005; 175:7791-7795.

35 Rosen DB, Bettadapura J, Alsharifi M, Mathew PA, Warren HS, Lanier LL: Cutting edge: lectin-like transcript-1 is a ligand for the inhibitory human NKR-P1A receptor. J Immunol 2005;175:7796-7799.

-36 Pozo D, Vales-Gomez M, Mavaddat N, Williamson SC, Chisholm SE, Reyburn H: CD161 (human NKR-P1A) signaling in NK cells involves the activation of acid sphingomyelinase. J Immunol 2006;176:2397-2406.

37 Mathew PA, Chuang SS, Vaidya SV, Kumaresan PR, Boles KS, Pham HT: The LLT1 receptor induces IFN-gamma production by human natural killer cells. Mol Immunol 2004; 40:1157-1163.

38 Roth P, Mittelbronn M, Wick W, Meyermann R, Tatagiba M, Weller M: Malignant glioma cells counteract antitumor immune responses through expression of lectin-like transcript-1. Cancer Res 2007;67:3540-3544.

39 Poggi A, Zocchi MR, Costa P, Ferrero E, Borsellino G, Placido R, Galgani S, Salvetti M, Gasperini C, Ristori G, Brosnan CF, Battistini L: IL-12-mediated NKRP1A up-regulation and consequent enhancement of endothelial transmigration of $\mathrm{V}$ delta $2+\mathrm{TCR}$ gamma delta+ T lymphocytes from healthy donors and multiple sclerosis patients. J Immunol 1999;162:4349-4354.

40 Roda-Navarro P, Arce I, Renedo M, Montgomery K, Kucherlapati R, Fernandez-Ruiz E: Human KLRF1, a novel member of the killer cell lectin-like receptor gene family: molecular characterization, genomic structure, physical mapping to the NK gene complex and expression analysis. Eur J Immunol 2000;30:568-576.

41 Vitale M, Falco M, Castriconi R, Parolini S, Zambello R, Semenzato G, Biassoni R, Bottino C, Moretta L, Moretta A: Identification of NKp80, a novel triggering molecule expressed by human NK cells. Eur J Immunol 2001:31:233-242.

-42 Welte S, Kuttruff S, Waldhauer I, Steinle A: Mutual activation of natural killer cells and monocytes mediated by NKp80-AICL interaction. Nat Immunol 2006; 7:1334-1342.

-43 Kuttruff S, Koch S, Kelp A, Pawelec G, Rammensee HG, Steinle A: NKp80 defines and stimulates a reactive subset of CD8 T cells. Blood 2008;113:358-369.

44 Biassoni R, Fogli M, Cantoni C, Costa P, Conte R, Koopman G, Cafaro A, Ensoli B, Moretta A, Moretta L, De Maria A: Molecular and functional characterization of NKG2D, NKp80, and NKG2C triggering NK cell receptors in rhesus and cynomolgus macaques: monitoring of NK cell function during simian HIV infection. J Immunol 2005; 174:5695-5705.
45 Mavilio D, Benjamin J, Kim D, Lombardo G, Daucher M, Kinter A, Nies-Kraske E, Marcenaro E, Moretta A, Fauci AS: Identification of NKG2A and NKp80 as specific natural killer cell markers in rhesus and pigtailed monkeys. Blood 2005;106:1718-1725.

46 Spreu J, Kuttruff S, Stejfova V, Dennehy KM, Schittek B, Steinle A: Interaction of C-type lectin-like receptors NKp65 and KACL facilitates dedicated immune recognition of human keratinocytes. Proc Natl Acad Sci USA 2010;107:5100-5105.

47 Fuller GL, Williams JA, Tomlinson MG, Eble JA, Hanna SL, Pohlmann S, Suzuki-Inoue K, Ozaki Y, Watson SP, Pearce AC: The C-type lectin receptors CLEC-2 and Dectin-1, but not DC-SIGN, signal via a novel YXXL-dependent signaling cascade. J Biol Chem 2007; 282:12397-12409.

48 Dennehy KM, Klimosch SN, Steinle A: Cutting edge: NKp80 uses an atypical hemiITAM to trigger NK cytotoxicity. J Immunol 2011;186:657-661.

49 Hamann J, Montgomery KT, Lau S, Kucherlapati R, van Lier RA: AICL: a new activation-induced antigen encoded by the human NK gene complex. Immunogenetics 1997;45: 295-300.

50 Pende D, Rivera P, Marcenaro S, Chang CC, Biassoni R, Conte R, Kubin M, Cosman D, Ferrone S, Moretta L, Moretta A: Major histocompatibility complex class I-related chain A and UL16-binding protein expression on tumor cell lines of different histotypes: analysis of tumor susceptibility to NKG2D-dependent natural killer cell cytotoxicity. Cancer Res 2002;62:6178-6186.

51 Akatsuka A, Ito M, Yamauchi C, Ochiai A, Yamamoto K, Matsumoto N: Tumor cells of non-hematopoietic and hematopoietic origins express activation-induced C-type lectin, the ligand for killer cell lectin-like receptor F1. Int Immunol 2010;22:783-790.

52 Kloss M, Decker P, Baltz KM, Baessler T, Jung G, Rammensee HG, Steinle A, Krusch M, Salih HR: Interaction of monocytes with NK cells upon Toll-like receptor-induced expression of the NKG2D ligand MICA. J Immunol 2008;181:6711-6719.

53 Thomas M, Boname JM, Field S, Nejentsev S, Salio M, Cerundolo V, Wills M, Lehner PJ: Down-regulation of NKG2D and NKp80 ligands by Kaposi's sarcoma-associated herpesvirus K5 protects against NK cell cytotoxicity. Proc Natl Acad Sci USA 2008; 105 : 1656-1661.

-54 Huarte E, Cubillos-Ruiz JR, Nesbeth YC, Scarlett UK, Martinez DG, Engle XA, Rigby WF, Pioli PA, Guyre PM, Conejo-Garcia JR: PILAR is a novel modulator of human T-cell expansion. Blood 2008;112:1259-1268.

55 Germain C, Bihl F, Zahn S, Poupon G, Dumaurier MJ, Rampanarivo HH, Berg PS, Spee P, Braud VM: Characterization of alternatively spliced transcript variants of CLEC2D gene. J Biol Chem 2010;85:3620736215. 